\title{
Ocorrência de Aedes (Stegomyia) aegypti (Linnaeus, 1762) (Insecta, Diptera, Culicidae) em bromélias, no município do Rio de Janeiro (Rio de Janeiro, Brasil)
}

\author{
Karen dos Santos Gonçalves ${ }^{1}$; Maria Conceição Messias ${ }^{2,3}$ \\ ${ }^{1}$ Escola Nacional de Saúde Pública Sérgio Arouca - ENSP, Fundação Oswaldo Cruz - FIOCRUZ, \\ Centro de Estudos de Saúde do Trabalhador Ecologia Humana-CESTEH, \\ Ambulatório de Pneumologia Ocupacional e Ambiental, Av. Leopoldo Bulhões, 4365, Manguinhos, \\ CEP 21045-900, Rio de Janeiro, RJ, Brasil \\ ${ }^{2}$ Departamento de Entomologia, Museu Nacional, Universidade Federal do Rio de Janeiro - UFRJ, \\ CEP 20940-040, Quinta da Boa Vista, São Cristóvão, Rio de Janeiro, RJ, Brasil \\ ${ }^{3}$ Autor para correspondência: Maria Conceição Messias, e-mail: mcmessias@terra.com.br
}

Gonçalves, K. S.; Messias, M. C. Occurrence of Aedes (Stegomyia) aegypti (Linnaeus, 1762) (Insecta, Diptera, Culicidae) in bromeliads in the municipality of Rio de Janeiro (Rio de Janeiro, Brazil). Biota Neotrop., vol. 8, no. 1, Jan./Mar. 2008. Available form: <http://www.biotaneotropica.org.br/v8n1/en/abstract?shortcommunication+bn01508012008>.

Abstract: Aedes (Stegomyia) aegypti (Linnaeus, 1762) (Insecta, Diptera, Culicidae) in bromeliads in municipality of Rio de Janeiro. Aedes (Stegomyia) aegypti (Linnaeus, 1762) (Insecta: Diptera, Culicidae) was found in bromeliad species at Fundação Oswaldo Cruz Campus, in the municipality of Rio de Janeiro (Rio de Janeiro, Brazil). Given the possibility of epidemiological impact, we emphasize the importance of studies that evaluate the Bromeliaceae as potential breeding sites in urban areas.

Keywords: Aedes breeding sites, bromeliads, urban areas.

Gonçalves, K. S.; Messias, M. C. Ocorrência de Aedes (Stegomyia) aegypti (Linnaeus, 1762) (Insecta, Diptera, Culicidae) em bromélias, no município do Rio de Janeiro (Rio de Janeiro, Brasil). Biota Neotrop., vol. 8, no. 1, jan./mar. 2008. Disponível em: <http://www.biotaneotropica.org.br/v8n1/pt/abstract?shortcommunication+bn01508012008>.

Resumo: Aedes (Stegomyia) aegypti (Linnaeus, 1762) (Insecta, Diptera, Culicidae) em bromélias, no município do Rio de Janeiro. Registra-se a ocorrência de Aedes (Stegomyia) aegypti (Linnaeus, 1762) em espécies de bromélias no Campus da Fundação Oswaldo Cruz (FIOCRUZ), no município do Rio de Janeiro (Rio de Janeiro, Brasil) e destaca-se a importância de novos estudos para se avaliar o significado epidemiológico das bromélias como criadouros urbanos potenciais.

Palavras-chave: criadouros, bromélias, áreas urbanas. 


\section{Introdução}

O objetivo deste trabalho é o de registrar a ocorrência de Aedes aegypti, principal vetor da dengue, em bromélias de uma área urbana.

A dengue é endêmica em todos os continentes, exceto na Europa (Forattini, 2000). Segundo Braga \& Valle (2007) a doença tem se destacado entre as enfermidades reemergentes e é considerada a mais importante das doenças virais transmitidas por artrópodos.

Consoli \& Lourenço-de-Oliveira (1994) afirmaram que raramente o Aedes aegypti utilizava as bromeliáceas como criadouros. Forattini (2000) relatou a coleta de Aedes aegypti em bromélias da espécie Aechmea fasciata (Lindley) fazendo referência à necessidade de reflexões epidemiológicas, tendo em vista o aumento da utilização de bromélias com finalidades decorativas.

Peryassú (1908) já havia registrado a ocorrência de Aedes aegypti em bromélias no município do Rio de Janeiro, e atualmente, elas podem ser observadas tanto no interior de residências, nos amplos terrenos dos condomínios fechados, nos jardins públicos, bem como nas calçadas bem cuidadas de avenidas.

\section{Material e Métodos}

A área de estudo foi o Campus da Fundação Oswaldo Cruz, no bairro de Manguinhos, localizado na zona norte do município do Rio de Janeiro, RJ. Foram delimitadas três áreas no Campus: a primeira, que corresponde à Portaria 01 - Av. Brasil, a segunda, o Jardim do Pavilhão Artur Neiva e a terceira, o Horto. Ao todo foram estudadas 75 bromélias pertencentes a 7 espécies distintas e 3 outras não identificadas a nível de espécie (Tabela 1).

As coletas foram realizadas, uma vez ao mês, no período de 03 de novembro de 2000 a 04 de dezembro de 2001. A água era retirada das bromélias, com o auxílio de um sifão adaptado, segundo Consoli \& Lourenço-de-Oliveira (1994). No mesmo dia de coleta o material era conduzido ao laboratório, onde realizava-se a triagem inicial, separando larvas e pupas e isolando-as na tentativa de eclosão do estágio adulto.

Larvas, pupas e adultos foram preparados entre lâminas e lamínulas, segundo Schlee (1968) e estudadas com a utilização de microscópio óptico. A identificação foi realizada de acordo com as chaves taxonômicas, segundo Forattini (2002). As bromélias foram identificadas pelo Dr. Gustavo Martinelli, do Instituto de Pesquisa Jardim Botânico do Rio de Janeiro.

\section{Resultados e Discussão}

Não foram registrados imaturos de Aedes aegypti nos meses de março, maio e agosto. O maior número de formas imaturas foi coletado nos meses de novembro de 2000 a janeiro de 2001em Alcantarea imperialis (Carrière). De 360 imaturos de Culicidae coletados, 294 eram Aedes aegypti (Tabela 1).

Cunha et al. (2002) também consideraram que as bromélias, que sofreram ação antrópica, deveriam ser estudadas como importantes criadouros de Aedes aegypti. Varejão et al. (2005) afirmaram ser necessário considerar a hipótese de que o A. aegypti poderia estar se adaptando às bromélias. Os autores, a partir de um levantamento de culicídeos realizado em cinco áreas da Cidade de Vitória, ES, registraram em quatro, a ocorrência de Aedes aegypti em bromélias próximas de prédios residenciais ou comerciais, sendo que cerca de $90 \%$ das bromélias pertenciam à espécie Alcantarea extensa. Das 115 formas imaturas coletadas, 20 pertenciam à espécie Aedes aegypti, em duas das cinco áreas investigadas.

Tabela 1. Abundância de Aedes aegypti coletados em bromélias no município do Rio de Janeiro, no período de novembro de 2000 a dezembro de 2001.

Table 1. Abundance of Aedes aegypti collected in bromeliads in the municipality of Rio de Janeiro, from november of 2000 to December of 2001.

\begin{tabular}{|c|c|c|c|}
\hline $\begin{array}{c}\text { Área } \\
\text { de Estudo }\end{array}$ & $\begin{array}{l}\text { Código de localização } \\
\text { da bromélia }\end{array}$ & Quantidade de Aedes aegypti & Espécies de Bromélias \\
\hline Jardim do Pavilhão & PAN-Gr01-Br01 & 9 & Alcantarea imperialis (Carrière) \\
\hline \multirow[t]{3}{*}{ Artur Neiva } & PAN-Gr01-Br02 & 172 & Alcantarea imperialis (Carrière) \\
\hline & PAN-Gr01-Br03 & 97 & Alcantarea imperialis (Carrière) \\
\hline & PAN-Gr06-Br01 & 3 & Neoregelia cf. coriacea (Antoine) \\
\hline Portaria & Port01-Gr01-Br01 & 1 & Neoregelia cf. coriacea (Antoine) \\
\hline \multirow[t]{13}{*}{01} & Port01-Gr01-Br02 & 6 & Neoregelia "hibrida" \\
\hline & Port01-Gr01-Br03 & 1 & Neoregelia cf. coriacea (Antoine) \\
\hline & Port01- Gr01-Br04 & 3 & Neoregelia cf. coriacea (Antoine) \\
\hline & Port01-Gr01-Br05 & 6 & Neoregelia cf. coriacea (Antoine) \\
\hline & Port01-Gr01-Br09 & 1 & Neoregelia cf. coriacea (Antoine) \\
\hline & Port01-Gr02-Br01 & 8 & Alcantarea imperialis (Carrière) \\
\hline & Port01-Gr04-Br03 & 12 & Vriesea sp. \\
\hline & Port01-Gr04-Br04 & 1 & Neoregelia cruenta (R. Graham) \\
\hline & Port01-Gr04-Br06 & 2 & Neoregelia cruenta (R. Graham) \\
\hline & Port01-Gr04-Br11 & 1 & Neoregelia cruenta (R. Graham) \\
\hline & Port01-Gr04-Br15 & 1 & Neoregelia cruenta (R. Graham) \\
\hline & Port01-Gr04-Br17 & 2 & Neoregelia cruenta (R. Graham) \\
\hline & Port01-Gr05-Br01 & 8 & Alcantarea imperialis (Carrière) \\
\hline \multirow[t]{5}{*}{ Horto } & Horto-Gr01-Br01 & 16 & Neoregelia cf. coriacea (Antoine) \\
\hline & Horto-Gr01-Br02 & 14 & Neoregelia compacta $(\mathrm{Mez})$ \\
\hline & Horto-Gr01-Br04 & 15 & Quesnelia sp. \\
\hline & Horto-Gr02-Br05d & 25 & Billbergia pyramidalis (Sims) \\
\hline & Horto-Gr02-Br01e & 24 & Billbergia pyramidalis (Sims) \\
\hline
\end{tabular}


Ocorrência de Aedes (Stegomyia) aegypti (Linnaeus, 1762) no Rio de Janeiro (Rio de Janeiro, Brasil)

Os resultados obtidos e os registros citados mostram que os tanques de bromélias necessitam ser estudados como um criadouro deste mosquito e avaliados sob o ponto de vista epidemiológico, considerando doenças como a dengue.

\section{Agradecimentos}

Ao Dr. Sebastião José de Oliveira, in memorian, pelos valiosos ensinamentos, ao Dr. Gustavo Martinelli do Instituto de Pesquisa Jardim Botânico pela identificação das espécies de bromélias, à Dra. Prof. Sonia Maria Lopes Fraga, do Departamento de Entomologia do Museu Nacional, UFRJ pelas sugestões e discussões, à CONCER Companhia de Concessão Rodoviária Juiz de Fora-Rio, pelo apoio.

\section{Referências Bibliográficas}

BRAGA, IMA APARECIDA E VALLE, DENISE. Aedes aegypti: histórico do controle no Brasil. Epidemiol. Serv. Saúde, jun. 2007, vol. 16, no. 2, p. 113-118.

CONSOLI, R. G. B. \& OLIVEIRA, R.L., 1994. Principais mosquitos de importância sanitária no Brasil. Rio de Janeiro. 228p.

CUNHA, S.P, ALVES J.R.C., LIMA, M.M., DUARTE, J.R., BARROS, L.C.V., DA SILVA, J.L, GAMMARO A.T, MONTEIRO FILHO, O.S, WANZELER, A. R. 2002. Presença de Aedes aegypti em Bromeliaceae e depósitos com plantas no Município do Rio de Janeiro, RJ. Rev. Saúde Pública. São Paulo. 36(2):244-5

FORATTINI, O.P. 2002. Culicidologia médica: identificação,biologia, epidemiologia. São Paulo: EDUSP, vol. 2.

FORATTINI, O.P., MARQUES, G.R.A.M. 2000.Nota sobre o encontro de Aedes aegypti em bromélias. Rev Saúde Pública; 37(6):729-734

PERYASSÚ, A.G. 1908. Os culicideos do Brasil. Instituto de Manguinhos, R.J. Brazil, 407p.

SCHLEE, D. 1968. Zur Präparation von Chironomiden. II. Die Behandlung ausgeblichenen bzw. getrockneten und das Reparieren schafter Präparate. Ann. Zool. Fenn., 5:127-129.

VAREJÃO, J.B.M., DOS SANTOS CLAUDINEY BIRAL, REZENDE H.R., BEVILACQUA, L. C., FALQUETO A. 2005. Criadouros de Aedes (Stegomyia) aegypti (Linnaeus, 1762) em bromélias nativas na Cidade de Vitória, ES. Revista da Sociedade Brasileira de Medicina Tropical 38(3):238-240 